\title{
Les objets intentionnels. À la frontière entre les actes et le monde
}

\author{
Maria Gyemant
}

L'année 1894 marque le début d'un des grands débats qui fondent la théorie de l'intentionnalité. Il s'agit, en effet, de l'année où sont écrits deux des textes essentiels sur le rapport que la conscience, sous la forme d'actes intentionnels, entretient avec l'objet. Le premier de ces textes est la thèse d'habilitation de Kasimir Twardowski, ancien élève de Franz Brentano, Sur la théorie du contenu et de l'objet des représentations. Le second est un texte d'Edmund Husserl, intitulé Objets intentionnels, qui n'a jamais atteint une forme finale, mais sur lequel Husserl est revenu à plusieurs reprises. Ce texte est une réponse directe et très critique au texte de Twardowski, réponse qui sera reprise dans une recension que Husserl en fait en 1896 et qui restera aussi hors publication. En se plaçant à des positions diamétralement opposées, les deux anciens élèves de Brentano s'inscrivent néanmoins dans une problématique ouverte par leur professeur et intrinsèque au concept même d'intentionnalité que celui-ci met en place. Il s'agit, en effet, dans les deux textes, d'aller au cœur de la question de l'intentionnalité, notamment de trouver une théorie qui puisse expliquer comment les actes subjectifs peuvent se rapporter à quelque chose d'objectif et de transcendant - comment, en d'autres termes, sortir de l'immanentisme que la conception brentanienne implique. 


\section{Les sources de la dispute}

La difficulté trouve son origine dans la définition même de l'intentionnalité telle qu'elle a été héritée de Brentano. Dans un passage très souvent cité de sa Psychologie du point de vue empirique, Brentano définit les phénomènes psychiques, en opposition avec les phénomènes physiques, comme étant caractérisés par «l'inexistence intentionnelle » de leur objet, autrement dit par le fait qu'ils se dirigent vers un objet qui leur est immanent (Brentano 124) ${ }^{1}$. Or, il y a dans cette définition une ambiguïté. Simplement dit, la définition de Brentano fait intervenir deux choses, un acte et un objet, sous un seul nom, celui d'intentionnalité. Cette ambiguïté ne passe pas inaperçue de Brentano lui-même qui, dans une note qui fait référence à ce passage, essaie d'expliquer ce que son concept d'« inexistence» signifie vraiment. Pourtant, cette note ne fait que témoigner de la difficulté sans parvenir à l'éclairer. Car Brentano affirme ici, en reprenant une thèse d'Aristote, qu'il y a une partie de l'objet, sa forme, qui est immanente, au sens propre du terme, à l'acte. Cependant, dans la même note il attire l'attention sur le fait que cette (in)existence ne doit pas être confondue avec l'existence proprement dite, et donc que l'objet n'est immanent à la conscience que dans un sens impropre du terme. L'objet est dit exister dans la conscience à la fois dans le sens propre et dans le sens impropre du terme. C'est précisément à partir de cette ambiguïté que Robin D. Rollinger identifiera deux directions dans l'école de Brentano, selon l'acception forte ou faible de l'immanentisme brentanien ${ }^{2}$. Les adeptes de l'immanentisme fort, Stumpf et Marty, soutiennent qu'une partie de l'objet existe réellement à l'intérieur de la conscience, tandis que l'immanentisme faible insiste sur la différence entre l'existence proprement dite des objets transcendants et l'existence seulement modifiée des objets immanents. C'est dans cette deuxième direction, selon Rollinger, que s'inscrit Twardowski, alors 
que Husserl rejettera toute forme d'immanentisme, qu'il soit fort ou faible, pour mettre en place sa propre conception de l'intentionnalité et de sa référencialité.

Twardowski et Husserl se situeront, tous les deux, par rapport à la thèse immanentiste de Brentano, dans une position relativement peu orthodoxe. Alors que Husserl critiquera énergiquement le psychologisme de Twardowski, nous devons remarquer avec Barry Smith que Twardowski est néanmoins responsable du premier pas vers la sortie du psychologisme, car il introduit pour la première fois dans un contexte psychologique la distinction entre le contenu et l'objet de la représentation ${ }^{3}$. Mais une fois cette distinction mise en place, une nouvelle série de questions s'ouvre à l'esprit. En effet, si Twardowski considère d'un côté le contenu immanent, et de l'autre l'«en face » de l'acte, l'objet réel, il devra ensuite rendre compte du rapport entre les deux et surtout du passage de l'un à l'autre. Car le risque que cette distinction engendre est de se trouver incapable d'expliquer le passage du contenu à l'objet et de se retrouver avec, d'un côté, le sujet qui a des actes, et de l'autre côté, des objets transcendants parfaitement indépendants de tout acte. C'est de cette difficulté que prennent naissance les « objets intentionnels » qui, dans une interprétation ou dans une autre, conservent leur caractéristique principale d'être des objets visés, et d'être tels qu'ils sont visés par une intention. Ces objets sont le signe distinctif de l'entre-deux où les actes viennent rencontrer la réalité transcendante pour la transposer dans l'immanence de la conscience.

Ce qui se cache, par conséquent, derrière la question du rapport intention-objet, est le problème plus essentiel de savoir comment articuler le logique et le psychologique, comment faire passer le monde dans le sujet sans lui créer un double psychologique. Alors que, dans sa thèse d'habilitation de 1894, Twardowski avancera comme réponse sa théorie du contenu immanent, présent dans toute représentation et qui se distingue de l'objet réel qui peut être 
confirmé ou infirmé par le jugement d'existence correspondant, Husserl hésitera toujours entre deux attitudes possibles. Une, plutôt négative, consistera dans la critique, parfois très virulente, de la conception twardowskienne du double mental, immanent, du monde. Cette critique, amorcée en 1894 et 1896, se retrouve en 1901 dans l'appendice aux $\S 11$ et 20 de la Recherche logique $V$. En ce qui concerne l'autre attitude, elle apparait dans une série de textes datant de la même période, où Husserl cherche à donner une solution propre à la question de savoir comment l'intention s'accroche à la réalité. Cette solution, qui passe par la théorie du remplissement, se retrouve dans Intuition et représentation, intention et remplissement de 1893 et dans les Études psychologiques de 1893-1894, les deux textes étant publiés dans le tome XXII de la Husserliana. La problématique du remplissement amorcée dans ces textes sera reprise dans la Sixième recherche logique dont elle constitue la thématique principale. Défini d'abord, dans les textes de 1894, en termes d'intérêt tendu et de satisfaction, le remplissement prend sa forme finale dans la Sixième recherche logique où il constitue l'acte de synthèse qui réunit une intention signitive avec une intention intuitive portant sur le même objet. L'intuition fournit à la simple signification le contenu intuitif qui re-présente (repräsentiert) l'objet visé et la relie ainsi au monde. Son degré de ressemblance à l'objet peut varier entre des limites très larges, tant qu'il fournit à l'intérêt tendu définissant l'intention un plus de satisfaction. Au cours du développement de cette théorie, le rapport entre intention et remplissement deviendra de plus en plus étroit, les deux arrivant, dans les tentatives de réécriture de la Sixième recherche logique de 1913-1914 (HUA XX), à se confondre et s'effacer sous un seul acte synthétique ${ }^{4}$.

La question brentanienne de l'immanence de l'objet se trouve, nous l'avons montré, au cœur du débat entre Husserl et Twardowski. Mais la source immédiate du conflit se retrouve dans le $\S 67$ de la Wissenschaftslehre de Bernard Bolzano. Dans ce texte, Bolzano traite de ce 
qu'il appelle des gegenstandlose Vorstellungen, des représentations sans objet. Une série d'exemples est invoquée ici pour illustrer ce genre spécial de représentations : le rien, les objets contradictoires comme le «cercle carré » et les objets fictifs probables (catégorie dans laquelle entrent tous les objets qui n'ont pas encore été découverts) et improbables (par exemple un centaure). Puisque, dans leur cas, les objets ne se retrouvent pas dans la réalité, alors que leurs représentations existent, Bolzano élabore sa théorie des représentations en soi, qui existent indépendamment de l'existence de leur référence, tout en se distinguant des contenus psychologiques de représentation. Ainsi, d'après Bolzano, la représentation fonctionne à deux niveaux : un niveau subjectif de la représentation effectivement eue par un sujet pensant, et un niveau objectif, idéal qui n'est rien d'autre que «la teneur sémantique de la représentation, et dont le modèle est clairement celui linguistique, d'unités de sens correspondant aux différents mots, même s'il demeure extrinsèque à ce sens d'être effectivement proféré ou non » (Benoist 17). Une représentation subjective aura toujours une représentation objective correspondante, tandis que la représentation objective peut exister indépendamment de son instanciation subjective. Ainsi, la théorie de Bolzano accommode facilement les représentations «sans objet » qui font partie de cette dernière catégorie de représentations en soi dépourvues de forme subjective $^{5}$. On remarque le fait que représentation sans objet ici signifie représentation sans objet effectif. Ainsi, une représentation dont l'objet est possible n'a pas plus d'objet qu'une représentation dont l'objet réunit des propriétés contradictoires. Il y a dans les deux cas une représentation objective, de même que, dans les deux cas, il n'y a, à proprement parler, pas d'objet. «La référencialité, ici, comme la non-référencialité, sont de fait» (25). Une représentation sans objet est une représentation qui n'est rien d'autre qu'une simple signification. La thèse bolzanienne est éminemment non intentionnaliste en cela qu'elle peut faire économie 
des représentations subjectives, se déployant uniquement sur le plan de l'idéalité des significations. Mais, comme le remarque Jocelyn Benoist, «il n’en reste pas moins que sa façon de poser le problème des "représentations sans objet" ouvre la voie à une distinction qui, retravaillée et interprétée en un sens différent, jouera un rôle majeur dans l'élaboration du point de vue intentionaliste » (20). Bolzano sera l'un des premiers à souligner le parallélisme entre le niveau du sens et celui de l'objet, ainsi que l'autonomie du premier par rapport au dernier, thèse qui sera au cœur de la conception husserlienne de l'intentionnalité.

Cependant, la caractéristique principale de la thèse bolzanienne est son antipsychologisme. En cela, elle entre en conflit avec la théorie brentanienne de l'intentionnalité, dont elle contredit l'axiome selon lequel tout acte, et donc tout acte de représentation aussi et principalement, doit avoir un objet. La mise face à face des deux conceptions débouche sur un paradoxe appelé à l'époque «le paradoxe des représentations sans objet». Il s'agit de tenir ensemble deux propositions concernant la représentation, qui engendrent en apparence un contresens :

1. Toute représentation a un objet.

2. Il y a des représentations dont l'objet n'existe pas.

L'entière construction twardowskienne s'efforcera de donner une solution à ce paradoxe. Se trouvant, en bon élève de Brentano, dans l'impossibilité de nier que toute représentation ait un objet, Twardowski essayera de relativiser le sens de l'objet et le sens de l'existence dans la deuxième proposition. Or, ce sera justement cette démarche qui suscitera une critique très énergique chez le jeune Husserl. Car accepter un régime différent d'existence pour tous les objets inexistants signifie mettre sur le même plan les fictions et les objets idéaux logiques et mathématiques. Husserl s'efforcera de sauver le statut objectif de ces objets que le 
dispositif twardowskien réduit à de simples fictions. Sa position se situe entre celles de Brentano et de Bolzano. L'idée de provenance bolzanienne d'une existence idéale des représentations, rejetée par Twardowski, sera récupérée par Husserl et intégrée à une construction intentionnelle inspirée par Brentano, sous la forme de la signification idéale qui constitue ce qui, dans la représentation, reste objectif et invariable.

\section{La solution de Twardowski}

Le texte Sur la théorie du contenu et de l'objet des représentations place Twardowski sur une position que Rollinger appellera «weak immanentism». Mais cette position installe néanmoins une distance par rapport à la conception brentanienne dont elle s'inspire. En effet, Brentano avait confondu objet et contenu de la représentation, ce qui a eu comme conséquence l'impossibilité de penser l'objet autrement que dans son immanence à une conscience. De son côté, Twardowski s'efforcera d'établir une distinction nette entre contenu et objet. Ainsi, sa position marque la sortie du psychologisme de Brentano, car elle fait justice au domaine des objets réels dans leur transcendance par rapport à la conscience.

L'enjeu du texte de Twardowski est donc de fixer des distinctions entre l'acte de représentation, son contenu immanent et l'objet transcendent. La première distinction, analysée par Twardowski dans le premier paragraphe de son texte, ne semble pas poser en soi beaucoup de problèmes. Il s'agit de distinguer un double sens dans l'expression « représentation ». Celle-ci nomme d'une part l'acte de représentation, le représenter, qui s'oppose au juger, au désirer, etc., et d'autre part, elle nomme le produit de cet acte, son contenu, autrement dit «le représenté ». Il est facile de distinguer entre l'acte et le contenu immanent à l'acte grâce à leur nature différente. La seule ambiguïté provient du fait que le même terme est utilisé pour les deux. Il s'agit donc 
d'une ambiguïté purement linguistique et extérieure à l'essence des termes. Un acte ne pourra pas être confondu avec son contenu parce que le contenu devient présent seulement par le moyen de l'acte. Bien sûr, dans une autre perspective, on pourrait affirmer aussi que l'acte devient présent au moyen de son contenu dont il est indissociable, qu'un acte sans contenu est impossible à saisir. Néanmoins, Twardowski pose cette distinction entre l'acte de représentation et le contenu représenté comme étant à peine problématique et il l'épuise en quelques phrases.

Il n'en va pas de même pour la distinction entre contenu et objet représenté. Cette distinction sera le fil conducteur de l'argumentation du texte de 1894 qui est dédié en totalité à son élucidation. Il s'agit ici aussi, au premier regard, d'une ambiguïté générée par le langage. En effet, le terme «représenté » peut être utilisé tantôt pour l'objet «existant en soi [an sich Bestehende] sur quoi se dirige notre représenter et notre juger », tantôt pour le contenu de la représentation, «1"“image" psychique existant "en" nous ("an" uns Bestehende) (Twardowski 88). Twardowski emploiera trois stratégies différentes pour mettre en évidence la différence entre contenu et objet. La première stratégie consiste à penser les représentations sur le modèle des noms. Un nom qui fonctionne normalement remplit trois tâches: il indique l'acte de représentation qui prend place dans la conscience du locuteur, il éveille un contenu dans la conscience de l'interlocuteur et il nomme un objet. Le nom a ainsi un rapport différent avec le contenu, pour lequel il constitue une sorte de support de transmission, et avec l'objet qu'il nomme. La deuxième stratégie sera d'analyser le terme «représenté » en tant qu'attribut appartenant d'un côté à l'objet, de l'autre au contenu. Cette fois, Twardowski jouera sur une distinction qui sépare les différents genres d'attributs en déterminants et modificateurs. Un attribut déterminant laissera l'objet auquel il s'applique inchangé. Il ne fera que renforcer l'identité et le degré de détermination de l'objet en question. Cependant, un attribut modificateur 
annulera tous les attributs déterminants qui définissent l'identité de l'objet et, par cela, l'objet même déterminé ainsi ne sera plus le même. Mais le même attribut peut jouer tantôt un rôle déterminant, tantôt un rôle modificateur. Ici, Twardowski a recours à l'exemple du tableau peint : l'attribut «peint» est déterminant pour le tableau, mais modificateur pour le paysage peint. Un paysage peint n'est plus un paysage du tout, mais, justement, un tableau. Cette ambivalence appartient aussi à l'attribut «représenté ». Twardowski en profitera pour mettre encore une fois en évidence la différence entre contenu et objet de la représentation. Ainsi, quand nous parlons d'«objet représenté » en ayant en vue le contenu de la représentation, l'attribut « représenté » sera utilisé dans un sens déterminant. Car il est tout à fait propre à un contenu d'être représenté : en effet, un contenu qui n'est pas représenté cesse complètement d'être un contenu. Quand, ensuite, nous appelons «objet représenté » l'objet qui se trouve en face de l'acte de représenter, le sens de l'attribut devient modificateur. L'objet n'est donc pas représenté dans le même sens que le contenu est représenté. Par conséquent, les deux ne se confondent pas.

Enfin, la troisième stratégie est plutôt un prolongement de son exemple antérieur qui met en évidence, cette fois, la relation que le contenu et l'objet entretiennent avec l'acte de représentation. L'exemple du tableau fournira l'outil pour faire cette différence. De même que l'image-tableau est un moyen par lequel le peintre rend le paysage, le contenu est un moyen pour rendre compte, dans la représentation, de son objet réel. «L'objet représenté », conclut ainsi Twardowski, en reprenant une distinction de Brentano,

c'est-à-dire l'objet sur lequel se dirige l'opération consistant à se représenter, l'acte de représentation, est l'objet primaire du fait de se représenter; le contenu par lequel l'objet devient représenté, est l'objet secondaire de l'opération consistant à représenter. (102) 
L'acte de représentation ne se rapporte pas de la même façon aux deux objets. L'objet primaire est le but de l'acte, tandis que l'objet secondaire est seulement le moyen pour atteindre ce but. Pour exprimer mieux cette idée, Twardowski reprend une distinction de Zimmermann qui affirme que l'objet est représenté par l'acte, tandis que le contenu est représenté dans l'acte. Cette distinction entre dans et par doit souligner le fait que le contenu se trouve à l'intérieur de la conscience, donc du même côté que l'acte, tandis que l'objet se trouve en relation, et par cela à l'extérieur de la conscience.

Dans une note qui fait référence à ce passage, Twardowski observe que la distinction entre l'objet primaire et l'objet secondaire se retrouve chez Brentano, mais dans un sens différent. Brentano inclut dans la catégorie de l'objet secondaire le tout de l'activité psychique, c'est-à-dire non seulement le contenu, mais aussi l'acte de représentation. Ainsi, chaque fois qu'il y a une représentation de quelque chose, il y aurait aussi une représentation de deuxième degré de l'acte de représentation même. Cette thèse sera rejetée par Husserl à plusieurs reprises par l'argument selon lequel une représentation qui constitue l'objet d'une autre représentation cesse de fonctionner elle-même comme acte de représentation. Une représentation, autrement dit, ne peut pas jouer à la fois le rôle d'acte et d'objet : si elle est considérée en tant qu'objet, son activité représentative devient bloquée. Quand Husserl critique Twardowski sur ce point, sa critique n'est pas entièrement juste, car Twardowski prend lui-même une distance envers la thèse brentanienne en excluant explicitement l'acte lui-même du domaine de l'objet secondaire.

Nous remarquons aussi que Twardowski distingue objet et contenu en identifiant la représentation d'abord avec la nomination, et ensuite avec la production d'images. Les deux exemples fonctionnent chez lui comme des analogies dont le résultat est que la signification est mise sur le même plan que l'image-copie. Le manque d'une véritable théorie de la signification 
suscitera de la part de Husserl une critique qui démontera point par point l'argumentation twardowskienne.

Les deux distinctions, entre acte et contenu et entre contenu et objet, s'appliquent sans difficulté là où les trois positions de l'acte, du contenu et de l'objet sont remplies et où la représentation fonctionne normalement. Mais qu'est-ce qui se passe quand la position de l'objet est vide, quand l'objet n'existe pas? Et surtout, quand il n'a aucune chance d'exister car ses propriétés sont contradictoires? Comment penser les représentations d'objets impossibles comme le « carré rond »? Twardowski rejettera explicitement l'idée bolzanienne des représentations sans objet. Et en effet, s'il l'acceptait, sa distinction entre contenu et objet serait largement relativisée. Tout l'effort argumentatif de Twardowski se concentre sur la distinction entre contenu et objet. Or, si un grand nombre de représentations n'a pas d'objet, sa distinction ne vaudrait que pour une seule classe de représentations, celles qui ont des objets réels, spatio-temporels. Twardowski essaiera donc de combattre l'idée de représentation sans objet en éliminant un par un les exemples que Bolzano fournit: le rien, les objets contradictoires et les fictions. Au $\S 5$ de sa Théorie du contenu et de l'objet des représentations, Twardowski prend position très clairement par rapport aux objets inexistants :

L'expression «représentation sans objet» est de telle sorte qu'elle contient une contradiction interne. Car il n'y a pas de représentation qui ne représenterait pas quelque chose en tant qu'objet; il ne peut pas y avoir de pareille représentation. Il y a, au contraire, de très nombreuses représentations dont l'objet n'existe pas. (114)

La solution twardowskienne garde donc le sens de la représentation relativement univoque en tant qu'acte de la conscience. Du côté du corrélat objectif de l'acte de représenter, Twardowski sera pourtant obligé d'introduire une série de distinctions qui servent à départager entre le contenu et l'objet de l'acte qui, dans ce cas, n'existe pas. Twardowski devra tenir compte 
des présupposés de son raisonnement : toute représentation devra avoir un objet, mais cet objet ne devra pas se confondre avec le contenu. La solution découle naturellement: il faudra interpréter l'objet de telle manière que, tout en manquant d'existence effective, il n'en sera pas moins objet de représentation. C'est de cette exigence que prennent naissance les objets intentionnels.

Pour réfuter la représentation sans objet de «rien », Twardowski exclura le « rien » de la sphère des objets en raison du fait qu'il est un terme syncatégorématique. Ainsi, dans son cas, il ne s'agirait pas d'une véritable représentation : parce que le « rien » ne fonctionne pas comme un nom, il doit apparaître en liaison avec un autre terme. Mais cette stratégie ne pourra pas s'appliquer aussi au cas des objets contradictoires. Quand l'objet de représentation est le carré rond, il y a bien un nom, le nom «carré rond ». Le nom remplit ses trois tâches, il indique un acte, communique un contenu et nomme un objet (108). La tentation est ici de dire que dans ce cas, le nom nomme le contenu et non l'objet. L'objet ne peut pas exister parce que ses déterminations s'annulent l'une l'autre, mais il y a un contenu qui remplace l'objet et auquel s'applique le nom. Twardowski prévient le lecteur contre cette confusion qui est combattue avec deux arguments. Le contenu et l'objet «carré rond» ne sont pas la même chose, parce que «premièrement, celui-ci existe, celui-là non; et deuxièmement, nous attribuons au nommé des propriétés qui se contredisent bien les unes les autres, mais qui ne reviennent certainement pas au contenu de représentation » (109). En effet, la propriété d'être carré et la propriété d'être rond ne sont pas des propriétés du contenu. Seulement un objet peut être carré ou rond, et s'il est les deux à la fois, il n'existe pas. Quand l'objet nommé « carré rond » devient ensuite sujet d'un jugement d'existence, il sera évidemment décrété par ce jugement comme inexistant. Mais si un jugement qui se construit sur la base de cette représentation aura la forme «Le carré rond n'existe pas », si 
donc la question de l'existence ne se pose qu'au niveau du jugement, il s'ensuit que «le carré rond » sera d'une certaine façon posé, affirmé par la représentation. Quelque chose doit être d'abord le support des propriétés contradictoires pour pouvoir ensuite être déclaré inexistant par le jugement. La représentation aura, autrement dit, toujours un objet. Mais cet «avoir» n'implique l'existence de l'objet que dans un sens modifié. «À l'existence effective d'un objet, telle qu'elle forme le contenu d'un jugement de reconnaissance, s'oppose l'existence phénoménale, intentionnelle de cet objet » (110). Or, cette existence intentionnelle n'est pas, à proprement dire, une existence.

La grande difficulté du texte de Twardowski est de maintenir toutes les distinctions qu'il introduit. En effet, le sens de l'objet intentionnel semble glisser sans cesse tantôt vers le contenu, tantôt vers l'objet. En dépit de l'insistance avec laquelle Twardowski rappelle que le contenu ne se confond pas avec l'objet — même dans le cas où celui-ci est seulement intentionnel — et que l'objet intentionnel, à son tour, n'est pas un objet « vrai », mais une sorte d'objet provisoire, nous rencontrons des difficultés à maintenir cette position. En ce qui concerne la distinction objet-contenu quand l'objet n'existe pas, la difficulté consiste en cela que l'objet intentionnel relève d'une dépendance par rapport à la conscience qui le rend en une certaine mesure immanent à celle-ci. En effet, il n'existe nulle part ailleurs. À ce sujet, il faudrait remarquer, comme le fait Jocelyn Benoist, que «l'immanence de l'objet, qui est immanence intentionnelle, ne doit pas être confondue avec une immanence réelle (celle du “contenu”) ». En effet,

[1]'objet en tant que visé et purement en tant que visé n'a pas l'existence, pas plus que l'inexistence : il est indifférent ontologiquement à de telles propriétés contrairement au « contenu », qui, quant à lui, renvoie à un morceau de réalité : la réalité mentale. (Benoist 92) 
En ce qui concerne la distinction entre l'objet intentionnel et l'objet vrai, elle semble valable dans la mesure où l'objet vrai existe dans le sens propre du mot, tandis que l'objet intentionnel n'existe que dans le sens modifié. En y regardant de plus près, la construction de Twardowski semble suggérer qu'il y a un seul type d'objet de représentation, alors que ce qui diffère est son mode d'existence, effectif ou modifié. Ainsi, un objet dont on peut dire qu'il est intentionnel n'aura jamais d'existence effective. Nous pouvons dire qu'il est intentionnel justement en vertu de ce manque d'existence proprement dite. Twardowski reprend de Brentano, d'après Jocelyn Benoist,

l'idée d'une existence intentionnelle, ou seulement «objective», qui est l'existence de l'objet en tant que seulement représenté, et doit être soigneusement distinguée de toute existence véritable ou effective, qui peut très bien manquer à l'objet - et dont l'imputation suppose toujours déjà la première forme d'existence («intentionnelle ») de l'objet. (90)

Or, si l'interprétation de Benoist est correcte, ce qui est notre conviction, alors nous ne voyons pas de raison pour limiter l'attribution de l'existence dans le sens modifié au seul cas où l'existence effective de l'objet sera imputée par le jugement correspondant. Si l'imputation de l'existence effective suppose toujours déjà l'existence intentionnelle de l'objet, la confirmation doit la supposer aussi. Or dans ce cas, la question de l'existence effective ne se pose vraiment qu'au niveau du jugement, le niveau de la représentation étant toujours marqué par l'existence provisoire, modifiée, de son objet. Twardowski le remarque aussi quand il observe que «la représentation aurait donc un objet aussi longtemps que ces contradictions ne deviendraient pas remarquées; mais quand, en un clin d'œil, celui qui se représente se mettrait à les apercevoir, la représentation cesserait d'avoir un objet » (Twardowski 110). Tout objet de représentation sera en attente de confirmation ou infirmation de son existence effective par un jugement correspondant. Ainsi, il faudra conclure que la théorie de Twardowski implique quatre et non 
trois termes qui entrent en relation : un acte de représentation, son contenu immanent, l'objet intentionnel et l'objet effectif qui n'entre en jeu qu'au niveau du jugement et qui peut être confirmé ou infirmé par celui-ci. La raison pour laquelle Twardowski ne remarque pas cette multiplication des éléments de l'équation est que l'objet effectif et l'objet intentionnel ne peuvent jamais être saisis à la fois. Si l'objet n'existe pas effectivement, alors, après coup, il faut conclure que la représentation a toujours eu seulement un objet intentionnel. Si, de l'autre côté, l'objet existe, l'objet intentionnel disparaît sous celui-ci. Mais ce qui est important ici est le fait que cette décision quant au type d'existence de l'objet de la représentation ne peut être prise qu'après coup, soit en passant par un jugement d'existence.

Toutes les différences semblent donc se jouer seulement au niveau du jugement d'existence. La représentation aura toujours la même forme qui relie un acte, un contenu et un objet intentionnel (que le jugement confirmera ou non). Elle aura toujours un objet qui doit, en un certain sens, exister. Or, ce sens est dépendent d'une conscience autant pour l'objet intentionnel que pour le contenu. La question de la vraie Existenz dans le sens fort, spatiotemporel, ne se pose qu'au niveau du jugement. En dépit des efforts d'argumentation de Twardowski et de son insistance sur la différence entre objet vrai, objet intentionnel et contenu de la représentation, nous ne pouvons pas ignorer les ambiguïtés qui naissent par l'introduction de cette différence entre objet effectif et objet intentionnel. Ce qui reste problématique est le statut ontologique des objets intentionnels qui ne sont pas immanents dans le même sens que le contenu, mais qui n'existent pas, non plus, dans le même sens que les objets confirmés par des jugements d'existence. En ce sens, la position de Twardowski a souvent été rapprochée de celle de Meinong, dans la conception duquel les objets couvrent des domaines bien plus étendus que celui du réel ${ }^{6}$. 


\section{La critique husserlienne du texte de Twardowski}

La critique de Husserl ira frontalement contre les deux acquis du texte twardowskien. Husserl attaquera d'abord la notion de contenu que Twardowski avait si méthodiquement mise en place, pour ensuite lancer ce qu'il appellera dans ses Leçons sur la doctrine de la signification de 1908 «la grosse artillerie » contre la distinction entre objets intentionnels et objets vrais. Il démontera ainsi, pas par pas, le dispositif twardowskien qui devait neutraliser le paradoxe des objets inexistants. Or, à la fin de cette démarche, Husserl retrouvera le paradoxe et devra en fournir une solution propre. Mais pourquoi Husserl refuserait-il la solution twardowskienne au paradoxe? L'enjeu de sa critique est, en effet, très précis et spécifique compte tenu de la direction que prennent ses recherches à l'époque. Si Husserl acceptait les objets immanents tels que Twardowski les présente, il serait obligé de ranger les objets mathématiques et, en général, tout objet idéal des sciences, dans la même catégorie que les objets fictionnels ou même les objets absurdes comme le «cercle carré ». Ils auraient tous, dans la représentation, le même statut d'objets intentionnels en attente d'un jugement d'existence qui les confirmera ou les infirmera selon qu'ils trouvent ou non, dans la réalité, un objet vrai correspondant. Or, il n'y a pas plus d'objets vrais dans le sens spatio-temporel correspondant à un objet idéal qu'à un objet fictionnel. Dans le dernier paragraphe de son texte, Twardowski affirme :

La représentation générale est toujours une représentation indirecte, non intuitive, et même non intuitive à un degré tel qu'elle a été tenue par beaucoup, à l'égal des représentations dont les objets montrent des marques distinctives contradictoires les unes avec les autres, pour tout à fait ineffectuable, et que son existence a été niée. (195)

Cette position a comme présupposé l'idée que la représentabilité d'un objet consiste en sa possibilité d'avoir une forme intuitive. Or, un objet général, tel un triangle qui ne serait ni isocèle, ni scalène, ni équilatéral, ne pourrait jamais prendre une forme intuitive. Un triangle 
intuitionné devra se ranger nécessairement dans l'une des trois catégories ${ }^{7}$. Les objets idéels resteraient, selon Twardowski, de simples objets intentionnels et leur conférer de l'existence signifierait s'exposer à la critique du platonisme.

Husserl ne pourra pas être d'accord avec une telle position. Elle impliquerait que les objets généraux n'existent qu'en vertu d'une conscience qui les penserait, à la manière des fictions. Pour sauver l'idéalité de ces objets, leur existence réelle, indépendante du sujet pensant, Husserl doit fournir une alternative à la conception, trop psychologiste, de Twardowski, et il le fera en introduisant une différence interne entre le côté logique, nécessaire et universel, et le côté psychologique contingent de l'acte de représentation. Pour Husserl, confondre le plan de l'image avec le plan de la signification est une «erreur fondamentale qui limite malheureusement beaucoup la valeur du mémoire concerné » (Objets intentionnels 349), car en ce qui concerne la signification, cette confusion lui ôte l'idéalité ${ }^{8}$, et quant au contenu-image, elle implique un dédoublement de l'objet par rapport à son statut d'existence. «Le dédoublement entre l'image et son objet (fût-il fictif) devient alors vecteur de l'intentionnalité et Twardowski réinterprète sa propre distinction entre contenu et objet dans des termes où le contenu est l'image » (Benoist 180).

La thèse qui constitue le présupposé du texte sur les Objets intentionnels, qui semble avoir été le thème d'un fragment précédant le texte et qui aujourd'hui n'existe plus, est qu'«à chaque représentation revient une teneur de signification»(Objets intentionnels 279). Ce présupposé témoigne de la position essentiellement antipsychologiste que Husserl adopte à cette époque. Sa critique impute à Twardowski le fait d'avoir traité la représentation seulement d'un point de vue psychologique, dans son effectivité, alors que Husserl, à la suite de Bolzano, opère une séparation entre deux niveaux de la représentation: le niveau psychologique, de l'acte 
concret, appartenant effectivement au sujet, et qui peut contenir des contenus intuitifs plus ou moins adéquats, et le niveau logique, de la représentation respective en tant qu'idéalité, ce qu'il y a de général dans tous les actes individuels qui peuvent représenter l'objet en cause. Le contenu de la représentation dans ce sens idéal est la signification. La signification est donc la seule partie nécessaire du contenu de représentation. «La signification », affirme Husserl, « est la seule détermination interne essentielle de la représentation» (314). Dans les Recherches logiques, cette thèse sera d'avantage approfondie. Le contenu idéal qui, dans tout acte intentionnel, et donc dans la représentation aussi, correspond à la signification de l'objet recevra alors le nom de « matière intentionnelle ». Le rôle de la matière sera d'indiquer à l'intérieur de l'acte quel est l'objet visé et en tant que quoi il est visé 9 .

Cette idée contredit la conception de Twardowski, dans laquelle c'est l'intuition qui constitue l'essence de la représentation. Pour soutenir ce point de vue, Twardowski fait référence à Aristote : «on ne peut jamais avoir une représentation non intuitive sans que celle-ci ne soit accompagnée d'une représentation intuitive (ou de plusieurs)» (Twardowski 197). Le caractère purement psychologique de cette thèse ne peut pas passer inaperçu : la représentation est conçue seulement comme un acte concret, qui fait jour dans la conscience d'un sujet pensant et qui, dans cette occurrence, prend appui sur des images surgies de l'expérience personnelle de ce sujet. «Les limites de la théorie de la représentation-image sont alors », remarque Jocelyn Benoist, «celles de l'intuition entendue comme intuition simple» (Benoist 184). La conséquence immédiate d'une telle position est un défaut de représentabilité des objets abstraits. Si n'est objet de représentation que ce dont on peut avoir une image, alors les objets mathématiques se placeront sur le même niveau que les objets contradictoires, car dans les deux cas une image correspondante serait impossible. Or, selon Husserl, ce n'est pas dans cette circonstance de la 
représentation que réside son essence, mais, justement, c'est en cela qu'une représentation reste la même dans une série d'instanciations psychologiques grâce à sa teneur idéale qui est la signification ${ }^{10}$. Et, par ce mouvement d'idéalisation, les objets généraux sont aussi légitimes que tous les autres objets de représentation.

La critique du psychologisme de Twardowski va donc dans deux directions. Premièrement, elle vise l'absence d'une vraie théorie de la signification chez Twardowski. «Twardowski identifie signification et représentation directement intuitive de l'objet signifié, tandis que le concept fondamental et seul déterminant au point de vue de la signification lui échappe complètement » (Recherche logique IV 88). Deuxièmement, il critique sous le titre de «solution populaire » une conception de la représentation comme image qu'il essaie clairement d'attacher à Twardowski. Or, si le texte twardowskien offre maints exemples qui soutiennent cette critique, il faudrait néanmoins lui rendre justice en notant les précautions que l'auteur prend par rapport à cette conception. En effet, au $\S 12$ de son texte, Twardowski remarque qu'une certain «psychologie primitive » aurait pu, à une époque, donner une telle réponse, mais qu'«il faille admettre, toutefois, une sorte de similitude photographique entre contenu et objet, ce semble être là, aujourd'hui, une question résolue en général dans le sens négatif » (Twardowski 154). Cependant, ces précautions sont infirmées non seulement par le fait que Twardowski explique le mécanisme de la représentation en ayant recours principalement à l'exemple du tableau, mais aussi, d'après Jocelyn Benoist, «Twardowski maintient cependant que, dans une certaine mesure, la constitution méréologique de l'objet peut et doit se refléter dans celle de la représentation. On retrouve donc ici, au niveau méréologique, une logique de l'image » (Benoist 182). Aux yeux de Husserl, après la confusion de la représentation avec la nomination effective, psychologique, qui passe à côté d'une vraie théorie de la signification comme entité idéale, la 
seconde analogie, qui rapproche la représentation au tableau, ne fait qu'approfondir l'erreur. Non seulement elle renvoie les objets généraux du côté de la fiction, comme nous l'avons expliqué plus haut, mais elle installe un dédoublement de l'objet et de ses modes d'existence qui sera inacceptable pour Husserl.

Nous rejoignons ainsi la deuxième et la plus importante critique que Husserl porte au texte de Twardowski, la critique de l'immanentisme. Nous avons fait appel dans notre texte au partage que Rollinger fait entre les élèves de Brentano qui, suite à sa thèse de l'inexistence intentionnelle, ont adopté un immanentisme fort, et ceux qui soutiennent un immanentisme faible. Husserl critiquera l'immanentisme et dans sa forme forte, et dans sa forme faible (Rollinger 248). L'argument que Husserl emploie contre l'immanentisme faible de Twardowski est que la distinction entre les objets existants et inexistants n'est pas une distinction entre des modes d'existence, mais entre des modes de représentation ${ }^{11}$.

Husserl reprend l'argument de Twardowski : toute représentation a un objet, mais certains objets ont une existence véritable, tandis que d'autres n'existent que de manière intentionnelle. «L'objet tout court et l'objet "pensé" », remarque Husserl non sans ironie, «cela fait deux »(Discussion 353). Ainsi, l'objet visé existe toujours là où il y a une représentation, mais l'objet lui-même peut parfois exister, parfois manquer. La question que Husserl pose alors est de savoir lequel des deux est l'objet sur lequel porte le jugement d'existence. Quand le jugement d'existence affirme que l'objet n'existe pas, il ne peut pas s'agir de l'objet intentionnel, qui appartient sans faute à toute représentation et, par cela, il existe, justement en tant que réalité psychologique.

Si l'objet «immanent» de la représentation (c'est ainsi qu'on dit carrément, au lieu d'objet «intentionnel ») est immanent au sens propre, alors son existence est aussi authentique et honorable que celle de la représentation elle-même, et il devient tout à 
fait impossible de comprendre pourquoi nous devons la regarder et la faire valoir en tant qu'elle serait simplement modifiée. (Objets intentionnels 286)

En effet, il semble que la distinction entre objet intentionnel et objet réel ne s'opère jamais, car au niveau de la représentation tous les objets existent seulement en tant qu'intentionnels, tandis que les jugements ne portent que sur les objets « vrais » qui sont les seuls qui peuvent exister ou ne pas exister. Les jugements ne porteraient alors pas sur les mêmes objets que les représentations. S'il y a un objet immanent même dans le cas ou le jugement pose sa nonexistence, alors les deux objets ne peuvent pas être les mêmes, car l'un existe et l'autre n'existe pas, ou existe seulement en un sens plus faible. S'ils ne sont pas les mêmes, alors le registre de la représentation et celui du jugement sont complètement déconnectés. Il résulte que la distinction entre objet intentionnel et objet vrai n'est que fonctionnelle, un simple artifice terminologique qui ne doit pas être pris au sens propre, qu'il

y a là seulement désignée une certaine fonction de toutes les représentations, à savoir celle qui consiste à intervenir, dans une connexion de jugement, d'une certaine façon, qui demeure typiquement la même, que le jugement d'existence correspondant soit ou non encore valable. (353)

Ainsi, selon Husserl, Twardowski confond les objets intentionnels avec le contenu qui, lui, ne manquerait jamais à la représentation. Mais le contenu est confondu à son tour avec l'image psychique qui n'est, en réalité, qu'une Repräsentation de l'objet et qui ne peut pas ainsi constituer le vrai objet visé par la représentation. En effet, Husserl conteste l'hypothèse d'un partage entre la conscience du sujet et le monde extérieur, l'objet intentionnel se trouvant « dans le sujet », l'objet vrai dans le monde. L'être-objet est parfaitement univoque, et déploie un seul et même plan dans lequel sont possibles existence et inexistence, sans que l'une nous oblige à faire retour vers un état inabouti ou diminué («mental») de l'objet (Benoist 187). L'objet vrai est selon lui toujours intentionnel, il est toujours objet justement dans sa relation avec une 
conscience. Autrement il ne serait pas question d'en parler. Chaque fois que l'on fait référence à un objet qui se trouve en face de nos actes subjectifs, l'objet est considéré précisément dans cette relation, comme objet de l'acte, et non dans son autonomie. Car un objet qui n'entre pas en relation avec une conscience par l'intermédiaire d'un acte est, pour cette conscience, comme inexistant. «Tout objet doit être considéré en tant qu'il est “intentionnel”, c'est-à-dire intentionné — et non spécialement immanent. Les "objets vrais" sont intentionnels comme les autres » (Benoist 204). Tout objet est donc intentionnel. Mais à la différence de Twardowski, pour Husserl l'objet intentionnel n'est pas immanent. Il ne loge pas dans l'acte, mais constitue le but vers lequel l'acte se dirige. Ainsi, la distinction entre objet vrai et objet intentionnel sera seulement une différence de statut logique. Le même objet sera visé, selon Husserl, tantôt par un acte de représentation, tantôt par un acte de jugement qui pose son existence. Les différences entre les objets ne seront plus intrinsèques aux objets mêmes, mais tiendront à la modalité par laquelle ils sont visés. Ainsi, l'objet reste partout univoque et identique à soi même.

Sur ce point, Benoist et Rollinger s'accordent pour attirer l'attention sur le fait que cette critique est en réalité une critique masquée de la thèse de Brentano, par rapport à laquelle Twardowski essaie lui-même de se détacher. En effet, Twardowski essaie d'éviter le dédoublement mental-extramental du lieu de l'objet en insistant sur la différence entre contenu et objet, soit-il inexistant dans le sens spatio-tempore ${ }^{12}$. «Le problème », remarque pourtant Benoist « est que Twardowski, s'il ne distingue pas deux lieux (mental et transcendant) de l'objet, distingue deux modalités d'existence du même objet. Et il est possible que, dans une telle distinction, il y ait comme un reliquat de la précédente » (Benoist 188). Pour Husserl, distinguer une existence effective et une existence modifiée serait une aberration, car elle ne fait que répéter avec d'autres mots le dédoublement de l'objet. En revanche, une distinction des actes de 
représentation, des modalités de la visée, expliquerait ce qui arrive là où l'objet de la représentation n'existe pas. Chaque fois qu'il y a visée, il y a visée de quelque chose. Mais cette chose peut être visée en tant qu'existante, ou en tant que simplement représentée, simplement intentionnée. Il n'y a pas, d'après l'argument de Husserl, des objets existants et des objets inexistants, de même que dans le monde ne se promènent pas à côté des lions déterminés des lions indéterminés. Si on peut parler d'un lion indéterminé, c'est parce que la façon de viser est indéterminée. Nous retrouvons ici le paradoxe des objets inexistants reformulé dans une terminologie intentionnaliste. Tout acte vise un objet intentionnel, mais certains actes visent des objets simplement intentionnels, autrement dit non existants. Husserl relativisera le sens de la proposition selon laquelle toute représentation aurait un objet. Cette manière de parler est considérée impropre, car les représentations ont toujours un objet seulement sous l'hypothèse que ces objets existent. Pourtant, il est possible de parler d'objets en mettant en relation certaines représentations avec d'autres représentations. Cette façon de juger a aussi valeur de vérité. Cela vaut même pour des jugements sur des objets contradictoires. Le fait de dire «Un carré rond est carré » est un jugement intrinsèquement vrai. Mais ici, le jugement fonctionne sous l'assomption que les objets qu'ils mettent en relation existent vraiment. Cette assomption n'est pas thématisée et le problème de l'existence ne se pose tout simplement pas. En effet, le jugement sur le carré rond devrait être reformulé ainsi : «Si un carré rond existe, il est carré ». La solution de Husserl au paradoxe des objets inexistants est de poser la question au niveau des actes et non des objets. Il y a des actes qui visent l'existence des objets et des actes qui visent les objets sous l'assomption d'existence, sans poser explicitement la question. La terminologie de l'assomption préfigure ce qui, dans les Recherches logiques, prendra la forme du partage des actes en positionnels et non positionnels. 
Ce mouvement, par lequel Husserl rabat du côté des actes la question de l'existence et de l'inexistence, va de pair avec une conception de l'objet qui n'est pas celle de Twardowski. Dans la conception husserlienne, l'objet est ce qui reste identique dans une multiplicité d'occurrences différentes. Cette identité se déploie aux deux niveaux qui se retrouvent aussi dans l'analyse du contenu : sur le plan subjectif, l'objet est ce qui reste identique à l'intersection d'une multiplicité d'actes subjectifs, «un point intentionnel d'unité dans une multiplicité illimitée d'actes possibles »; sur le plan objectif, «l'objet est une unité idéale, eu égard [...] à l'infinité des vérités qui valent pour lui » (Objets intentionnels 319). Vus ainsi, les objets contradictoires seront compris à partir du cas «normal » des objets existants : «ils ne peuvent exister que pour ainsi dire parodiquement, dans la forme apparente du système de validité — et de validation qui est celui des objets légitimes mêmes »(Benoist 205). Si un objet est ce qui reste identique sur le plan subjectif, à l'intersection de tous les actes qui portent sur lui, et sur le plan objectif, à l'intersection de toutes les vérités qui sont valables pour lui, alors un objet contradictoire sera un (pseudo) objet en tant qu'il sera maintenu en tant qu'identique à lui-même par les actes subjectifs portant sur lui. En ce qui concerne le plan objectif, il se dissipera à cause du fait qu'il ne fonde aucune vérité. Ainsi, un objet fictif ou contradictoire sera objet (simplement intentionnel) seulement dans la mesure où il est dépendant d'une subjectivité, tandis que les objets vrais, les objets mathématiques inclus, garderont leur identité idéale indépendamment du fait d'être ou non pensés par une subjectivité. 


\section{Conclusion}

La solution que Husserl propose au paradoxe des objets inexistants s'oppose à celle de Twardowski, qui soutenait comme vrai le fait que toute représentation a un objet. Pourtant, l'opposition des deux démarches est seulement apparente. En fait, elles sont très semblables en ce qui concerne la stratégie. Car le but final des deux démarches est de conserver les deux propositions du paradoxe. Pour cela, la stratégie employée est de tenir pour vraie l'une des deux propositions, Twardowski la première, Husserl la deuxième, et d'essayer une interprétation relativisant les termes de l'autre, afin de l'intégrer dans une conception unitaire.

Pour résoudre le paradoxe, Twardowski avait multiplié les sens de l'objet et de l'être, en donnant ainsi un statut spécial à tout objet qui n'a pas d'existence effective. En faisant cela, il rangeait les objets idéaux dans la même catégorie que les objets fictifs et les objets contradictoires. Husserl combattra cette position dans le souci de sauver le statut nécessaire des objets idéaux logiques et mathématiques qui ne doivent en aucun cas varier d'un sujet à l'autre. Ainsi, il emploiera, pour résoudre le paradoxe, une autre stratégie : il élargira et le sens de l'objet, et le sens de l'être, pour accommoder les objets idéaux, mais en évitant de faire, comme Twardowski, des distinctions à l'intérieur de ce domaine ${ }^{13}$. Désormais, sera objet tout ce qui peut être nommé et sera existant tout objet qui peut fonder des énoncés vrais. De cette façon, Husserl réussit à partager entre les objets fictifs et contradictoires, qui peuvent être nommés mais ne fondent jamais des jugements d'existence vrais, et les objets idéaux mathématiques qui, eux, se trouvent entièrement dans le domaine du vrai.

En dépit de la critique systématique que Husserl fait de chaque point fort de l'argumentation de Twardowski, l'importance du texte Sur la théorie du contenu et de l'objet des représentations reste indéniable. Nous serons sur ce point d'accord avec Jocelyn Benoist en 
affirmant qu' «il a eu un rôle décisif pour le développement de la phénoménologie, puisque c'est en réaction par rapport à lui que Husserl a formé sa propre théorie et a été conduit à inventer une solution proprement phénoménologique du problème » (Benoist 70).

\section{Notes}

${ }^{1}$ Cf. Barry Smith, «Logic and the Sachverhalt»: «When Brentano talks of “objects", he is not referring to putative transcendent targets of mental acts. [...] Brentano is referring, rather, to immanent "objects of thought", and in fact no distinction is drawn in Brentano's treatment in Psychology between "content" and "object" in this sense » (article consulté sur Internet à l'adresse http://ontology.buffalo.edu/smith/articles/logsvh.html, le $1^{\text {er }}$ avril 2009).

2 "We can distinguish between strong immanentism (according to which the object actually, albeit partly, exists in consciousness) and weak immanentism (according to which the object exists only in a modified sense)»(Rollinger 247).

${ }^{3}$ Cf. Barry Smith, Austrian Philosophy: «Where Brentano had seen content and object as effectively one and the same, Twardowski regarded the content as a mental "picture" or "image" of the object of the act. Every act, according to Twardowski, has both a content and an object, though the object of an act need not in every case exist » (157).

${ }^{4}$ Il serait intéressant de mettre en rapport ces deux attitudes que Husserl adopte par rapport à l'intentionnalité entre 1893 et 1913. Cela d'autant plus que, si en 1894 Husserl critique Twardowski en offrant en revanche la solution du remplissement, en 1913, dans Ideen 1, il ne reste de cette critique qu'une simple différence de degré. La démarche de Twardowski apparait comme «une formulation anticipée [...] de la "réduction" » qui manque pourtant de radicalité car elle opère «un seul pas en arrière par rapport à la "transcendance objective", sans oser revenir jusqu'aux "actes" eux-mêmes, entièrement absents en effet de son ouvrage, à part la place purement nominale qui leur y est réservée au début » (English 16). Comment se produit ce changement d'attitude envers Twardowski et qu'est-ce qu'il reste de la théorie du remplissement dans le contexte de la réduction transcendantale, cela constituerait un bon sujet de recherche qui, pourtant, dépasse l'ambition de notre présent propos.

5 «Toute représentation subjective contient comme son sens (sa "matière": Stoff) une représentation objective, qui, en raison même de son statut sémantique, a une dimension d'idéalité, et est indépendante des fluctuations de la subjectivité représentante » (Benoist 18).

${ }^{6}$ Rollinger, 155-208. 
${ }^{7}$ Barry Smith commente là-dessus: «There are, on Twardowski's view, a number of important similarities between general objects and objects with contradictory characteristics. The former may, indeed, be counted as special cases of the latter (if it is true that there is something contradictory about a triangular figure that is neither equilateral nor isosceles nor scalene). Both general objects and contradictory objects are capable of being presented only nonintuitively and indirectly. And both, according to Twardowski, are such that, in and of themselves, they do not exist » (Austrian Philosophy 167).

${ }^{8}$ «It is to be noted here that the meaning of a name is identified by Twardowski with the content of a presentation, and that this content is moreover called "psychical" (for it is, after all, something which exists in the subject just as much as the act itself does). The resulting view that meanings are psychical entities is of course totally unacceptable to Husserl » (Rollinger 141).

${ }^{9}$ Edmund Husserl, $5^{\mathrm{e}}$ Recherche logique, ch. III.

${ }^{10}$ Cf. Barry Smith, «Logic and the Sachverhalt»: «Husserl's theory has built into its very foundations the idea of a parallelism of structure between (1) immanent contents on the level of our empirically executed acts and (2) ideal contents on the level of logic. He is thereby able to account in a very natural way for the fact that the laws of logic apply to actual thinkings, speaking and inferrings, while at the same time doing justice to the necessity which accrues to such laws by virtue of the fact that they relate primarily to a certain ideal or universal species and only secondarily to the immanent contents by which these species may come to be instantiated».

${ }^{11}$ «This division - just like the division of objects into determinate and indeterminate ones and also the division of them into possible and impossible ones - is however derived from a division among presentations » (Rollinger 248).

12 "Husserl's attack is directed against the notion of an immanent object, a notion which he clearly tries to pin on Twardowski. We must however be careful not to misinterpret Twardowski's position, for we have seen that he maintains that the object which does not exist is not to be identified with the content, not with that which is, properly speaking, immanent in consciousness. The content exists in the strict and proper sense, whereas the intentional existence of the object in the cases of the kind under consideration exists in a modified sense; that is to say, it does not exist at all »(Rollinger 149).

${ }^{13}$ « Reste que Husserl a bien, au passage, posé un sens élargi de l'existence, qui est un sens propre, et qui semble pouvoir prendre en charge ce qu'on nomme ordinairement "existence mathématique" $[\ldots]$ et que le problème de la référence aux objets mathématiques en tant qu'objets formels [...] nous conduit à cette zone-limite où, d'une certaine façon, le sens propre et le sens impropre de l'existence se confondent. Dans la mesure où ce qui importe, ce sont les enchainements déductifs et le type de contraintes propres qu'ils exercent sur l'objet, le régime assomptif est ici celui de la vérité même » (Benoist 212). 


\section{Ouvrages cités}

Benoist, Jocelyn. Représentation sans objet. Paris: P.U.F., 2001.

Bolzano, Bernard. Wissenschaftslehre. Stuttgart: Friedrich Frommann Verlag, 1987.

Brentano, Franz. Psychologie vom empirischen Standpunkt. Leipzig: Felix Meiner Verlag, 1924.

English, Jacques. «Présentation ». Husserl-Twardowski. Sur les objets intentionnels (18931901). Paris: Vrin, 1993: 9-84.

Husserl, Edmund. «Discussion de K. Twardowski (1896)». Dans Husserl-Twardowski. Sur les objets intentionnels (1893-1901). Trad. J. English. Paris: Vrin, 1993: 349-356.

—. «Objets intentionnels (1894)». Dans Husserl-Twardowski. Sur les objets intentionnels (1893-1901). Trad. J. English. Paris: Vrin, 1993: 277-326.

-. Recherches logiques. Tome second, $2^{\mathrm{e}}$ partie. Trad. H. Elie, A. L. Kelkel et R. Scherer. Paris: P.U.F., 1961.

Rollinger, Robin D. Husserl's Position in the School of Brentano. Dordrecht/Boston/London: Kluwer Academic Publishers, 1999.

Smith, Barry. Austrian Philosophy. The Legacy of Franz Brentano. Chicago/LaSalle: Open Court Publishing Company, 1994.

—. «Logic and the Sachverhalt». The Monist, vol. 72, n 1 (1989): $52-69$ (consulté sur Internet à l'adresse http://ontology.buffalo.edu/smith/articles/logsvh.html).

Twardowski, Kasimir. "Sur la théorie du contenu et de l'objet des représentations (1894)». Dans Husserl-Twardowski. Sur les objets intentionnels (1893-1901). Trad. J. English. Paris: Vrin, 1993: 85-200. 